\title{
Analysis of Highway Sloped Median Performance for Containment of Errant Vehicles
}

\author{
Oluremi Ayotunde Olatunbosun'1, Rui He², Olurotimi Shitta-Bey ${ }^{3}$ \\ ${ }^{1}$ School of Engineering, University of Birmingham, Birmingham, UK \\ ${ }^{2}$ Department of Mechanical Engineering, Virginia Polytechnic Institute and State University, Blacksburg, VA, USA \\ ${ }^{3}$ Department of Mechanical Engineering, University of Lagos, Lagos, Nigeria \\ Email: o.a.olatunbosun@bham.ac.uk
}

How to cite this paper: Olatunbosun, O.A., He, R. and Shitta-Bey, O. (2018) Analysis of Highway Sloped Median Performance for Containment of Errant Vehicles. World Journal of Engineering and Technology, 6, 68-80.

https://doi.org/10.4236/wjet.2018.61004

Received: November 27, 2017

Accepted: January 29, 2018

Published: February 1, 2018

Copyright (c) 2018 by authors and Scientific Research Publishing Inc. This work is licensed under the Creative Commons Attribution International License (CC BY 4.0).

http://creativecommons.org/licenses/by/4.0/

\begin{abstract}
Errant vehicles occur as a result of the driver losing control of the vehicle. This may be due to sudden illness, dozing off or skidding while attempting a manoeuvre. In containing such an errant vehicle on a highway, the priority is to avoid collision with other vehicles. A sloped highway median provides a run-off area for such vehicles where the vehicle can be slowed down and stopped without the danger of being re-directed into the path of other vehicles as may occur with edge barriers. Here, the effect of a containment barrier at the bottom of the sloped median is studied with a view to prevent the vehicle from being redirected outside the median after colliding with the barrier. The focus of this work is on the change of kinematic states due to the collision, so a momentum-based vehicle collision analysis is developed, with the collision energy loss related to the vehicle stiffness being considered by coefficient of restitution. The average maximum lateral displacements post-collision are read from the diagram of vehicle $x-y$ trajectories. In this way, the most suitable median slope 1:6 is selected.
\end{abstract}

\section{Keywords}

Vehicle Collision Model, Simulation, Highway Sloped Median

\section{Introduction}

A sloped highway median provides a run-off area for errant vehicles where they can be slowed down and stopped without the danger of being re-directed into the path of other vehicles as may occur with edge barriers. According to Gabler 
et al. [1], cross-median accidents are the most dangerous type of highway crashes so they are usually prevented by placement of a containment barrier at the bottom of the slope to prevent the errant vehicle from crossing the median and running into the opposite carriageway. However, possible redirection of the vehicle colliding with the barrier, back to the same carriageway might cause accidents as serious as the fatal cross median crash.

The research on Highway Median Safety in recent years, with wide application of simulation software, has mainly focused on two themes: one is investigation of double-sloped median terrain's influence on vehicle behaviour [2]; the other is design of barrier, placed in the median, to improve the performance of the median [3]. In 2008, a systematic method, VDA, Vehicle Dynamics Analyses [4] was proposed by the National Crash Analysis Center (NCAC), which plotted the trajectory of a vehicle driving into the median in terms of vertical and lateral position. Based on VDA, further research used both Finite Element Analysis (FEA) software and Multi-body Dynamics software to simulate a vehicle driving into the median, from which the guideline of optimal median barrier placement was drafted [5]. A trade-off was found by Stine et al. between designing a median to prevent vehicular rollover and designing it for preventing median crossovers, after a total run of 111,132 simulations in CarSim $^{\mathrm{TM}}$ [6]. Reliability of applying CarSim $^{\mathrm{TM}}$ in such research was validated by Uzunsoy et al. [7] by comparing the result of simulation with that of physical off-road tests.

The results of these researches indicate that in the study of errant vehicle behaviour in a double sloped median, the profile and geometric dimension of the median play decisive roles, and using simulation software of either FEA or Multi-body Dynamics is effective. The focus of this paper is to determine the most suitable median slope to prevent the vehicle from being re-directed outside the median after collision with the containment barrier. A time-saving method is proposed to simulate the kinematic change of the errant vehicle during collision with the containment barrier while $\operatorname{CarSim}^{\mathrm{TM}}$ is used to simulate the vehicle's trajectory before and after the collision.

\section{Methodology}

CarSim $^{\mathrm{TM}}$ provides a reliable simulation environment for the dynamics of an errant vehicle as its non-linear Multi-Body Dynamics vehicle model has been shown to reflect the vehicle dynamics property of real vehicles running on the sloped terrain surface of a road median [7]. However, it cannot simulate the collision with the containment barrier. Therefore additional software has been adopted in simulating the collision phase of the errant vehicle behaviour.

\subsection{Vehicle Simulation Structure}

Various types of mathematical vehicle collision model have been developed by researchers for two purposes, vehicle collision accident reconstruction, and vehicle post-collision active safety control strategy development [8] [9] [10] [11]. 
Brach developed a 3 Degree of Freedom (DOF) collision model based on planar collision mechanics, in which the collision force does not need to be calculated [8]. A similar collision model, based on momentum expression of Newton's $2^{\text {nd }}$ laws, has been employed in the software PC-crash which specialises in vehicle collision simulation or reconstruction [8] [11]. In contrast, another vehicle collision software EDSMAC 4, is based on a different collision model which calculates the collision force by using crash stiffness and acceleration expression of Newton's $2^{\text {nd }}$ law [10] [12] [13]. To develop active safety strategy after light impacts, Zhou et al. developed a 4 DOF collision model including roll motion and tyre forces which are not considered by Brach [8] [9]. The successful development of these mathematical collision models, especially the momentum-based model, demonstrates that vehicle kinematic behaviour right after the collision could be calculated by using these models.

In this study, the kinematic change of the vehicle during the collision with the rigid containment barrier is approximated by using the collision model based on planar impact mechanics. Thus a hybrid simulation platform is used consisting of a multi-body dynamics simulation for the pre- and post-collision phases (using $\operatorname{CarSim}^{\mathrm{TM}}$ ), and planar impact mechanics (using $M A T L A B^{\mathrm{TM}}$ ). The general structure of the simulation, shown in Figure 1, consists of three stages, Simulation of Vehicle Motion before Collision, Computation of Collision Model for Vehicle and Rigid Barrier, and Simulation of Vehicle Motion after Collision.

\subsection{Collision Model for Vehicle and Rigid Barrier}

The collision model based on planar impact mechanics developed by R.M. Brach [8] was chosen to calculate the post-collision velocities of vehicles since Car$\operatorname{Sim}^{\mathrm{TM}}$ is incapable of simulating the collision of the vehicle with rigid barrier. Such momentum-based collision model has already been applied in the commercial software PC-crash [14] which specializes in the simulation of vehicle-barrier

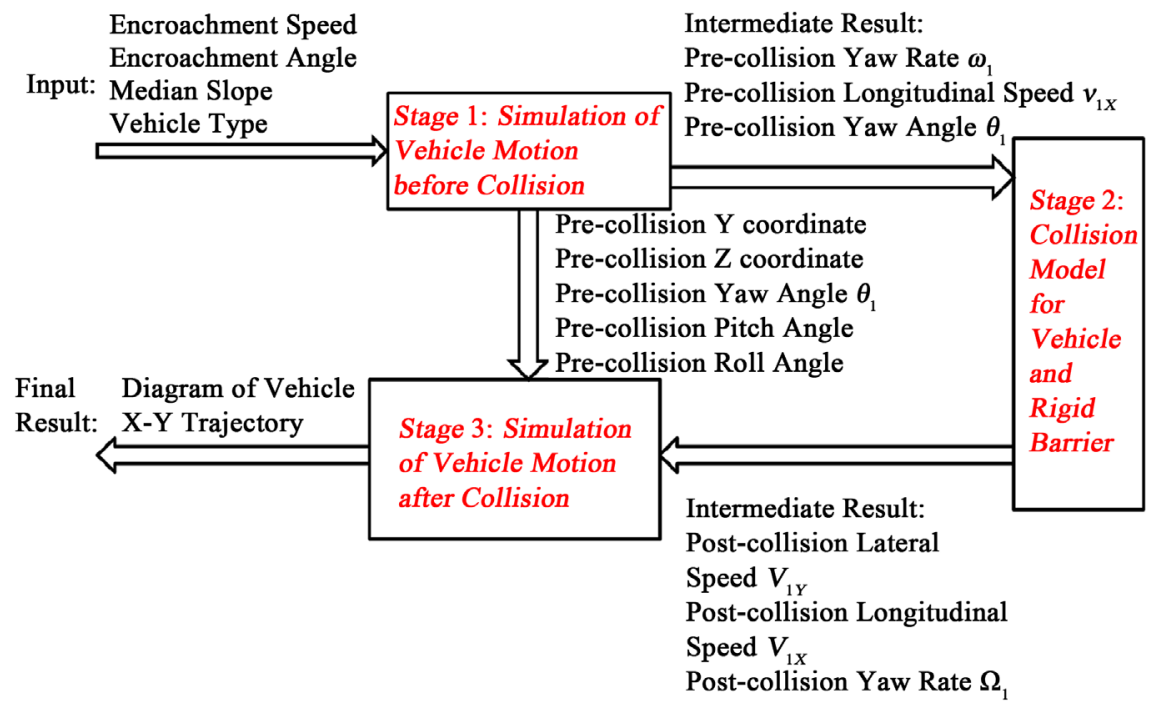

Figure 1. General structure of simulation. 
and vehicle-vehicle collision [15]. An alternative would have been Finite Element Analysis (FEA) but it is too time-consuming [16] for this project which requires at least 36 runs of simulation. An alternative vehicle collision model for the software EDSMAC 4, which computes collision force in order to obtain acceleration and velocity by using Newton's $2^{\text {nd }}$ law [12], was disregarded because the collision force is not of interest.

The following assumptions have been established for the application of the collision model:

1) The plane on which the vehicle is running (motion plane) just before the collision and the motion plane right after the collision are assumed to be the same.

2) The common contact surface (Figure 2) is parallel to the $x$ coordinate, and the right front wheel centre is on that surface.

3) The level of force acting over the common contact surface is significantly higher than other forces, such as aerodynamic force, tire-roadway friction, etc. So impulses of all forces other than contact forces are neglected [8].

4) The duration of the contact force impulse is very short, which implies that during contact, accelerations are high to the extent that velocities change suddenly and displacements (changes in position and orientation) are negligible [8]. Therefore, the final position and orientation in the pre-collision simulation could be assumed to be the initial position and orientation in the post-collision simulation.

5) Though the deformation during the collision is inevitable, the value of mass and moments of inertia about the $Z$ coordinate, and the position of the sprung mass centre relative to the vehicle are assumed to be unchanged.

6) Coefficient of restitution $e$ is assumed to be in the range of $0.1-0.3$ [14].

7) Larger absolute values of pre-collision yaw-angle (collision angle), produce more severe inelastic deformation of the vehicle, which leads to a smaller value of $e$ [17].

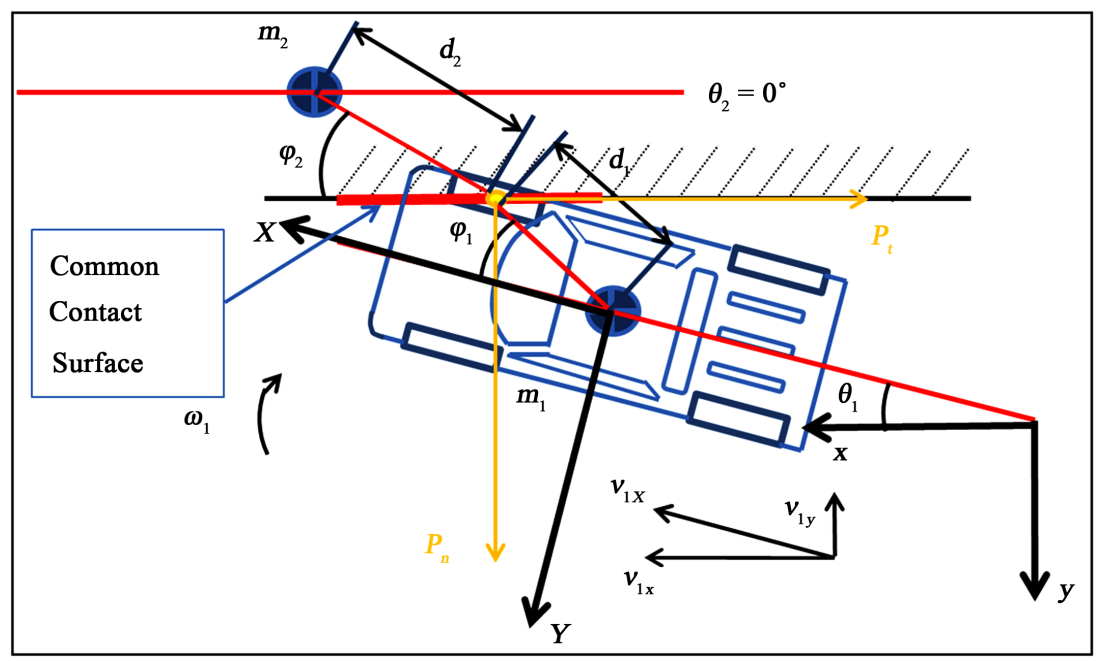

Figure 2. Free-body diagram of vehicle-barrier collision. 
8) Impulse ratio $\mu$ can be approximated by an equivalent coefficient of friction. Since it cannot be determined experimentally or estimated by means such as analytical modelling of the mechanism of tangential force generation, it is taken as the coefficient of dynamic friction between the common materials of the barrier and car body for approximation [8] [18].

Based on Assumption 1, planar impact mechanics including six pre-collision vehicle kinematic states $\left(v_{1 x}, v_{1 y}, \omega_{1}, v_{2 x}, v_{2 y}, \omega_{2}\right)$ is applied to the collision model. These represent the pre-collision linear and rotational velocities of the vehicle and barrier in the earth-fixed coordinates.

The first three variables are known from the pre-collision simulation result (see Stage 1 in Figure 1), and the last three variables are assumed to be 0 for the barrier. Also according to that simulation result, other kinematic variables such as vehicle pitch rate and roll rate could be assumed to be negligible. Correspondingly, eight post-collision vehicle motion variables $\left(V_{1 x}, V_{1 y}, \Omega_{1}, V_{2 x}, V_{2 y}, \Omega_{2}, P_{t}, P_{n}\right)$ are to be calculated by using Equations (1)-(11). These variables represent the post-collision linear and rotational velocities of the vehicle and barrier in the earth-fixed coordinates as well as the tangential impulse $\left(P_{t}\right)$ and normal impulse $\left(P_{n}\right)$.

Newton's laws in the form of impulse and momentum are applied to the bodies as shown in Figure 2. The equations of impulse and momentum for vehicle and barrier in $x$ coordinate and $y$ coordinate are:

$$
\begin{gathered}
m_{1}\left(V_{1 x}-v_{1 x}\right)=P_{t} \\
m_{2}\left(V_{2 x}-v_{2 x}\right)=-P_{t} \\
m_{1}\left(V_{1 y}-v_{1 y}\right)=P_{n} \\
m_{2}\left(V_{2 y}-v_{2 y}\right)=-P_{n}
\end{gathered}
$$

According to Newton's second law, changes in angular momentum are equal to the moments of the impulses on each body. By taking moments of momentum about vehicle mass centre, two more equations about vehicle yaw rates before and after the collision are obtained:

$$
\begin{gathered}
I_{z 1} \cdot\left(\Omega_{1}-\omega_{1}\right)=-d_{1} \cos \left(\varphi_{1}+\theta_{1}\right) P_{n}+d_{1} \sin \left(\varphi_{1}+\theta_{1}\right) P_{t} \\
I_{z 2} \cdot\left(\Omega_{2}-\omega_{2}\right)=-d_{2} \cos \left(\varphi_{2}+\theta_{2}\right) P_{n}+d_{2} \sin \left(\varphi_{2}+\theta_{2}\right) P_{t}
\end{gathered}
$$

Coefficient of restitution $e$ is defined by Equation (7) [18], and the higher the value of $e$, the more elastic will be the collision [17].

$$
e=-\frac{\text { Relative Rebound Velocity Normal to the Contact Plane }}{\text { Relative Approach Velocity Normal to the Contact Plane }}
$$

Equation (8) is obtained by applying Equation (7) to the vehicle and barrier in Figure 2. The relative normal velocities, $v_{c r n}$ and $V_{C r}$ (before and after collision) could be obtained by using the rigid-body kinematic relationship between the contact point and mass centre, as shown in Equation (9) and (10). 


$$
\begin{gathered}
e=-\frac{V_{C r n}}{v_{c r n}} \\
V_{C r n}=\Omega_{1} d_{1} \cos \left(\varphi_{1}+\theta_{1}\right)+V_{1 y}+\Omega_{2} d_{2} \cos \left(\varphi_{2}+\theta_{2}\right)-V_{2 y} \\
v_{c r n}=\omega_{1} d_{1} \cos \left(\varphi_{1}+\theta_{1}\right)+v_{1 y}+\omega_{2} d_{2} \cos \left(\varphi_{2}+\theta_{2}\right)-v_{2 y}
\end{gathered}
$$

Collecting and manipulating Equations (1)-(10), and transforming them into matrix form leads to Equation (11). The values of known variables in Equation (11) are obtained from Vehicle Assembly Screen in $\operatorname{CarSim}^{T M}$ : From Assumption 2, $m_{1}$ equals to the summation of sprung mass and unsprung mass; $I_{z 1}$ could be read directly; $d_{1}$ and $\varphi_{1}$ are calculated by Pythagorean theorem given the front track width and the horizontal distance between the wheel centre and mass centre; $m_{2}(\mathrm{~kg})$ and $I_{z 2}\left(\mathrm{~kg} \cdot \mathrm{m}^{2}\right)$ are assigned values of $10^{7} ; d_{2}$ shares the same value as $d_{1}$, so do the $\varphi_{2}$ and $\varphi_{1}$, which treats the barrier as a vehicle of the same size as, but far heavier than, the bullet vehicle [8]. The values of $v_{2 x}$, $v_{2 y}$ and $\omega_{2}$ are taken as zero, while $v_{1 x}, v_{1 y} \omega_{1}$ and $\theta_{1}$ are known from the pre-collision simulation result. $\theta_{2}$ equals 0 as shown in Figure 2. With the values of known variables substituted, post-collision vehicle motion variables could be computed from Equation (11).

$$
\begin{aligned}
& {\left[\begin{array}{cccccccc}
-m_{1} & 0 & 0 & 0 & 0 & 0 & 0 & 0 \\
0 & -m_{2} & 0 & 0 & 0 & 0 & 0 & 0 \\
0 & 0 & -m_{1} & 0 & 0 & 0 & 0 & -1 \\
0 & 0 & 0 & -m_{2} & 0 & 0 & 0 & 1 \\
0 & 0 & 0 & 0 & -I_{z 1} & 0 & d_{1} \cos \left(\varphi_{1}+\theta_{1}\right) & -d_{1} \cos \left(\varphi_{1}+\theta_{1}\right) \\
0 & 0 & 0 & 0 & 0 & -I_{z 2} & d_{2} \cos \left(\varphi_{2}+\theta_{2}\right) & -d_{2} \cos \left(\varphi_{2}+\theta_{2}\right) \\
0 & 0 & 1 & -1 & d_{1} \cos \left(\varphi_{1}+\theta_{1}\right) & d_{2} \cos \left(\varphi_{2}+\theta_{2}\right) & 0 & 0 \\
0 & 0 & 0 & 0 & 0 & 0 & 1 & -\mu
\end{array}\right] \cdot\left[\begin{array}{c}
V_{1 x} \\
V_{2 x} \\
V_{1 y} \\
V_{2 y} \\
\Omega_{1} \\
\Omega_{2} \\
P_{t} \\
P_{n}
\end{array}\right]} \\
& =\left[\begin{array}{cccccccc}
-m_{1} & 0 & 0 & 0 & 0 & 0 & 0 & 0 \\
0 & -m_{2} & 0 & 0 & 0 & 0 & 0 & 0 \\
0 & 0 & -m_{1} & 0 & 0 & 0 & 0 & 0 \\
0 & 0 & 0 & -m_{2} & 0 & 0 & 0 & 0 \\
0 & 0 & 0 & 0 & -I_{z 1} & 0 & 0 & 0 \\
0 & 0 & 0 & 0 & 0 & -I_{z 2} & 0 & 0 \\
0 & 0 & e & -e & -e d_{1} \cos \left(\varphi_{1}+\theta_{1}\right) & -e d_{2} \cos \left(\varphi_{2}+\theta_{2}\right) & 0 & 0 \\
0 & 0 & 0 & 0 & 0 & 0 & 0 & 0
\end{array}\right] \cdot\left[\begin{array}{c}
v_{1 x} \\
v_{2 x} \\
v_{1 y} \\
v_{2 y} \\
\omega_{1} \\
\omega_{2} \\
0 \\
0
\end{array}\right]
\end{aligned}
$$

Impulse ratio $\mu$ defined by Equation (12) measures how much tangential impulse is generated during the collision [17] [18].

$$
\mu=\frac{P_{t}}{P_{n}}
$$

An improper value of $\mu$ would produce unrealistic results of post-collision vehicle motion variables contrary to physical laws. Therefore, two criteria are imposed to validate the computation result.

The first criterion is to calculate the kinetic energy of the vehicle before and after the collision by using Equations (13) and (14). If the kinetic energy after the 
collision becomes higher than that before the collision, then the result of computation by using Equation (6) will be invalid.

$$
\begin{aligned}
& T_{1_{\text {pre-collision }}}=\frac{1}{2} \times m_{1}\left(v_{1 x}^{2}+v_{2 x}^{2}\right)+\frac{1}{2} \times I_{z 1} \omega_{1}^{2} \\
& T_{1_{\text {post-collision }}}=\frac{1}{2} \times m_{1}\left(V_{1 x}^{2}+V_{2 x}^{2}\right)+\frac{1}{2} \times I_{z 1} \Omega_{1}^{2}
\end{aligned}
$$

The second criterion is to check that the impulse ratio assumed is less than the critical impulse ratio $\mu_{0}$ when the vehicle and barrier reach a common tangential velocity-that is Equation (15). The vehicle is assumed to continue to slide over the contact surface throughout the contact duration, so the ratio of $\mu$ to the critical impulse $\mu_{0}$ must be lower than 1 [8].

$$
V_{1 x}-V_{2 x}-d_{1} \sin \left(\varphi_{1}+\theta_{1}\right) \Omega_{1}-d_{2} \sin \left(\varphi_{2}+\theta_{2}\right) \Omega_{2}=0
$$

Assembling Equations (1)-(13) into matrix form as shown in Equation (16) and using the definition of impulse ratio, it is possible to calculate $\mu_{0}$. If the ratio of $\mu$ to $\mu_{0}$ is larger than 1 , then the post-collision motion vector computed by Equation (11) will be invalid.

$$
\begin{aligned}
& {\left[\begin{array}{cccc}
-m_{1} & 0 & 0 & 0 \\
0 & -m_{2} & 0 & 0 \\
0 & 0 & -m_{1} & 0 \\
0 & 0 & 0 & -m_{2} \\
0 & 0 & 0 & 0 \\
0 & 0 & 0 & 0 \\
0 & 0 & 1 & -1 \\
1 & -1 & 0 & 0 \\
-m_{1} & 0 & 0 & 0 \\
0 & -m_{2} & 0 & 0 \\
0 & 0 & -m_{1} & 0 \\
0 & 0 & 0 & -m_{2} \\
0 & 0 & 0 & 0 \\
0 & 0 & 0 & 0 \\
0 & 0 & -e & e \\
0 & 0 & 0 & 0
\end{array}\right.} \\
& =\left[\begin{array}{cccc}
0 \\
0
\end{array}\right.
\end{aligned}
$$

$\begin{array}{cc}0 & 0 \\ 0 & 0 \\ 0 & 0 \\ 0 & 0 \\ -I_{z 1} & 0 \\ 0 & -I_{z 2} \\ \left(\varphi_{1}+\theta_{1}\right) & d_{2} \cos \left(\varphi_{2}+\theta_{2}\right) \\ \sin \left(\varphi_{1}+\theta_{1}\right) & -d_{2} \sin \left(\varphi_{2}+\theta_{2}\right)\end{array}$

$$
\left.\begin{array}{cc}
0 & 0 \\
0 & 0 \\
0 & -1 \\
0 & 1 \\
d_{1} \sin \left(\varphi_{1}+\theta_{1}\right) & -d_{1} \cos \left(\varphi_{1}+\theta_{1}\right) \\
d_{2} \sin \left(\varphi_{2}+\theta_{2}\right) & -d_{2} \cos \left(\varphi_{2}+\theta_{2}\right) \\
0 & 0 \\
0 & 0
\end{array}\right] \cdot\left[\begin{array}{c}
V_{1 x} \\
V_{2 x} \\
V_{1 y} \\
V_{2 y} \\
\Omega_{1} \\
\Omega_{2} \\
P_{t} \\
P_{n}
\end{array}\right]
$$

$$
\left.\begin{array}{cccc}
0 & 0 & 0 & 0 \\
0 & 0 & 0 & 0 \\
0 & 0 & 0 & 0 \\
0 & 0 & 0 & 0 \\
-I_{z 1} & 0 & 0 & 0 \\
0 & -I_{z 2} & 0 & 0 \\
-e d_{1} \cos \left(\varphi_{1}+\theta_{1}\right) & -e d_{2} \cos \left(\varphi_{2}+\theta_{2}\right) & 0 & 0 \\
0 & 0 & 0 & 0
\end{array}\right] \cdot\left[\begin{array}{c}
v_{1 x} \\
v_{2 x} \\
v_{1 y} \\
v_{2 y} \\
\omega_{1} \\
\omega_{2} \\
0 \\
0
\end{array}\right]
$$

The values of $V_{1 x}$ and $V_{1 y}$ are substituted into Equations (17) and (18) and resolved into the longitudinal speed $V_{1 X}$ and lateral speed $V_{1 Y}$ of the vehicle (vehicle-fixed coordinates) after the collision, based on Assumption 4. The values of $V_{1 X}, V_{1 Y}$ and $\Omega_{1}$ calculated by Equations (11), (17) and (18) and validated by Equations (13), (14) and (16) are input to the simulation of vehicle motion after collision (post-collision vehicle motion).

$$
\begin{gathered}
V_{1 X}=V_{1 x} \cos \theta_{1}+V_{1 y} \sin \theta_{1} \\
V_{1 Y}=V_{1 x} \sin \theta_{1}-V_{1 y} \cos \theta_{1}
\end{gathered}
$$

Equations (1)-(18) are implemented as $M A T L A B^{T M} \mathrm{M}$-files for the computation of the collision model. 


\subsection{Simulation Procedure}

$\operatorname{CarSim}^{\mathrm{TM}}$ is applied to simulate the vehicle motion before collision (pre-collision simulation): the vehicle is running at specified encroachment speed and encroachment angle before passing the median border; when the vehicle just passes the median border, all the control actions exerted on the vehicle are released, resulting in a control-free vehicle whose motion status is only subject to environmental factors such as gravity and terrain; the simulation is terminated instantly the vehicle contacts the rigid barrier. These conditions are implemented in $\operatorname{CarSim}^{\mathrm{TM}}$ as VS commands. At this point the coordinates of the vehicle in the earth-fixed coordinates $(x, y, z)$ are recovered as well as the vehicle's dynamic state (velocities, orientation etc.) in the vehicle-fixed coordinates $(X, Y, Z)$. The vehicle-fixed coordinate system is a moving coordinate system which is fixed to the centre of gravity of the vehicle whereas the earth-fixed coordinate system is a coordinate system whose origin is permanently fixed to the earth (see Figure 2). The vehicle's pre-collision kinematic state is converted into the earth fixed coordinates $\left(V_{1 x}, V_{1 p}, \omega_{1}\right)$ and used as input into the collision model to compute the post-collision kinematic state. The resulting post-collision kinematic state ( $V_{1 x}$, $\left.V_{1 p}, \Omega_{1}\right)$ is then transformed into the vehicle-fixed coordinates $\left(V_{1 X}, V_{1 Y}, \Omega_{1}\right)$. These, along with the pre-collision coordinates constitute the initial state of the vehicle for the post-collision simulation in $\mathrm{CarSim}^{\mathrm{TM}}$. Again, all controls are released for the post-collision simulation. The post-collision simulation needs to end when the vehicle just runs out of the median or contacts the barrier again. These conditions are again implemented in CarSim $^{\mathrm{TM}}$ as VS commands.

\section{Results and Discussion}

\subsection{Simulation Parameters}

In this study, the effect of median slope on the effectiveness of the containment barrier has been investigated. Median slopes of 1:4, 1:5, and 1:6 [6] were investigated. In order to manifest the effect of only the median slope, other geometric parameters, e.g., median width and median depth were kept constant. The median width was assigned a value of 75 feet $(22.86 \mathrm{~m})$, an average value of median widths applied in rural areas of some states in the USA [19]. A median depth of 6.25 feet $(1.905 \mathrm{~m})$ was assigned, the very value for the median profile of 1:6 median slope when its bottom width equals zero. A general view of the road layout with median and containment barrier is shown in Figure 3.

The encroachment angles used were $5^{\circ}, 10^{\circ}$, and $15^{\circ}$ and the encroachment speed was chosen to be $37.55 \mathrm{mph}$ (about $60 \mathrm{~km} / \mathrm{h}$ ). These values are representative of the values of typical vehicle encroachment parameters. Encroachment angles smaller than $15^{\circ}$ cover $75 \%$ of vehicle encroachment accident cases [20]. Encroachment angles larger than $15^{\circ}$ are more inclined to cause severe collisions, which indicate a higher energy loss [18] and less possibility that the vehicle runs back to the carriageway. The value of the chosen encroachment speed, $60 \mathrm{~km} / \mathrm{h}$, is the mean value of the maximum and minimum of the encroachment 


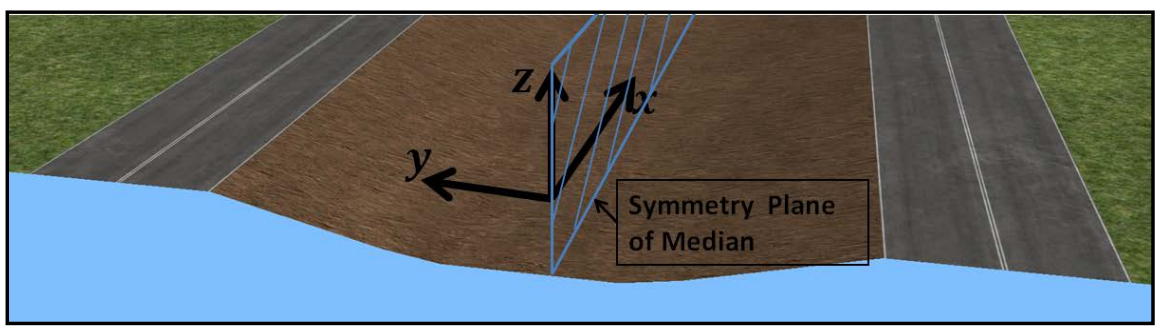

Figure 3. General view of median profile (Median slope 1:4).

speed interval $35.1 \mathrm{mph}$ to $40 \mathrm{mph}$, which accounts for the largest proportion of the relevant traffic accidents (35\%) among other intervals [21]. Other values of encroachment speed are not considered, as the vehicle at a speed lower than 60 $\mathrm{km} / \mathrm{h}$ would not travel back to the carriageway if it is contained in the median at an encroachment speed of $60 \mathrm{~km} / \mathrm{h}$; and an encroachment speed much higher than $60 \mathrm{~km} / \mathrm{h}$ would result in an severely inelastic collision or fly over the median barrier [22], such phenomena are beyond the scope of this study.

The vehicle encroachment simulation tests were carried out for four classes of vehicles, taken from the CarSim $^{\mathrm{TM}}$ database, namely: C-Class Hatchback 2012, D-Class Sedan, E-Class Sedan and Pickup. These represent a wide range of existing vehicle product, such as Audi A3, Ford Focus, Chevrolet Silverado, BMW 3 Series, BMW 5-Series, etc [23]. All the default configurations of the vehicle models in $\operatorname{CarSim}^{\mathrm{TM}}$ were kept.

From the result of pre-collision simulation, the collision angle $\theta_{1}$ is relatively small, ranging from $15^{\circ}$ to $25^{\circ}$. Correspondingly, the vehicle velocity, normal to the contact surface (Figure 2), ranges from $4.31 \mathrm{~km} / \mathrm{h}$ to $7.04 \mathrm{~km} / \mathrm{h}$. For such range of normal speed during the collision, the value of $e$ is between 0.25 and 0.3 [17], falling in the range of $e$ specified in Assumption 6. Therefore for approximation, and based on Assumption 7, the following discrete function is applied:

$$
e= \begin{cases}0.30, & \theta_{1} \in[15,20] \\ 0.25, & \theta_{1} \in(20,25]\end{cases}
$$

As described in Assumption 8, the value of $\mu$ could be related to the coefficient of friction when no experimental value is available. For approximation, the value of 0.3 is assumed in this study based on some sliding friction coefficient data [24].

\subsection{Intermediate Results}

The intermediate result refers to the kinematic variables (see Figure 1) of the pre-collision simulation and computation of collision model, some of which are input to the next stage of the method so that the final result of the whole simulation could be calculated stage by stage. Examples of intermediate results derived from the raw data obtained after the computation of the collision model are shown in Table 1 for two of the vehicles:

The first two derived variables are metrics for validating the vehicle-barrier collision. The results show that in all cases, the imposed criteria for validating 
Table 1. Data of derived kinematic variables for vehicle (a) Class C HATCHBACK; and (b) Pick up, full size.

(a)

\begin{tabular}{|c|c|c|c|c|c|c|c|c|c|}
\hline \multirow{2}{*}{ Class C Hatchback } & \multicolumn{9}{|c|}{ Median Slope } \\
\hline & \multicolumn{3}{|c|}{$1: 4$} & \multicolumn{3}{|c|}{$1: 5$} & \multicolumn{3}{|c|}{$1: 6$} \\
\hline Encroachment angle (deg) & 5 & 10 & 15 & 5 & 10 & 15 & 5 & 10 & 15 \\
\hline Rate of kinematic energy loss & 0.2755 & 0.3126 & 0.3766 & 0.2976 & 0.3360 & 0.3923 & 0.2800 & 0.3134 & 0.3700 \\
\hline Ratio of $\mu$ to $\mu_{0}$ & 0.2854 & 0.3029 & 0.3376 & 0.2957 & 0.3162 & 0.3478 & 0.2885 & 0.3045 & 0.3354 \\
\hline Increase rate of yaw angle magnitude & 2.7925 & 1.0975 & 0.5882 & 3.0280 & 1.1897 & 0.6255 & 2.7946 & 1.0796 & 0.5570 \\
\hline Change rate of longitudinal speed & 0.0196 & 0.0230 & 0.0265 & 0.0175 & 0.0223 & 0.0258 & 0.0094 & 0.0155 & 0.0191 \\
\hline Change of roll angle (deg) & -0.3013 & -0.4550 & -0.5899 & 0.8173 & 2.0116 & 2.1755 & 8.9402 & 8.8387 & 8.6747 \\
\hline
\end{tabular}

(b)

\begin{tabular}{|c|c|c|c|c|c|c|c|c|c|}
\hline \multirow{2}{*}{ Pick Up, Full Size } & \multicolumn{9}{|c|}{ Median Slope } \\
\hline & \multicolumn{3}{|c|}{$1: 4$} & \multicolumn{3}{|c|}{$1: 5$} & \multicolumn{3}{|c|}{$1: 6$} \\
\hline Encroachment angle (deg) & 5 & 10 & 15 & 5 & 10 & 15 & 5 & 10 & 15 \\
\hline Rate of kinematic energy loss & 0.2842 & 0.3149 & 0.3706 & 0.2808 & 0.3095 & 0.3625 & 0.2645 & 0.2950 & 0.3510 \\
\hline Ratio of $\mu$ to $\mu_{0}$ & 0.2439 & 0.2568 & 0.2840 & 0.2425 & 0.2543 & 0.2797 & 0.2348 & 0.2477 & 0.2746 \\
\hline Increase rate of yaw angle magnitude & 2.9116 & 1.1294 & 0.5912 & 2.8646 & 1.1015 & 0.5700 & 2.6795 & 1.0188 & 0.5253 \\
\hline Change rate of longitudinal speed & 0.0336 & 0.0363 & 0.0379 & 0.0309 & 0.0341 & 0.0355 & 0.0238 & 0.0280 & 0.0305 \\
\hline Change of roll angle (deg) & -0.4441 & -0.7045 & -1.0522 & 3.9291 & 4.7617 & 5.4827 & 9.0980 & 9.0561 & 8.7428 \\
\hline
\end{tabular}

the simulation results are satisfied i.e. in terms of the loss in kinetic energy and ratio of the assumed impulse ratio to the critical impulse ratio i.e. $\left(\mu / \mu_{0}\right)$.

The last three variables compare the vehicle kinematic status post-collision with that of the initial condition for the pre-collision simulation. The important observations from these results are:

- As the encroachment angle increases (collision angle increases too), the rate of kinematic energy loss increases, accounted for by the fact that a larger collision angle results in an increased inelastic deformation.

- As the vehicle drives downward the sloped terrain, the yaw angle can increase considerably (up to $290 \%$ in case of the $5^{\circ}$ encroachment angle). However the sloped terrain has little effect on the longitudinal speed.

- The most notable effect is on the roll angle where there is a change of up to $900 \%$ for the 1:6 median slope which is quite substantial, whereas for the flat bottomed 1:4 slope, the change is quite modest. The indication is that the flat bottom enables the suspension to level before the collision, resulting in less roll on impact.

\subsection{Final Results}

The $\operatorname{CarSim}^{\mathrm{TM}}$ simulation of the post-collision trajectory of the vehicle allows an assessment to be made of the susceptibility of the vehicle to be re-directed out- 
side the median into the path of other vehicles. Figure 4 shows the trajectories of the Class $C$ Hatchback after collision for the three median slopes (1:4, 1:5 and $1: 6)$ at three encroachment angles $\left(5^{\circ}, 10^{\circ}\right.$ and $\left.15^{\circ}\right)$. The $1: 4$ median slope results in, by far, the largest rebound, while there is very little difference between the 1:5 and 1:6 median slope results. Nevertheless, in all cases, the vehicle remains well within the median slope boundary.

The post-collision trajectories for all four vehicles tested on the three median slopes at the same three encroachment angles described earlier are shown in Figure 5. A summary of the results is presented in Table 2 which clearly indicates that the 1:6 median slope out-performs the other two median slopes in terms of susceptibility of the vehicle to be re-directed outside the road median. The vehicle motion envelope for the 1:6 median is shown in Figure 5. This indicates that in most instances the vehicle will not deviate far from the median centre line also reducing the susceptibility to roll over [25].

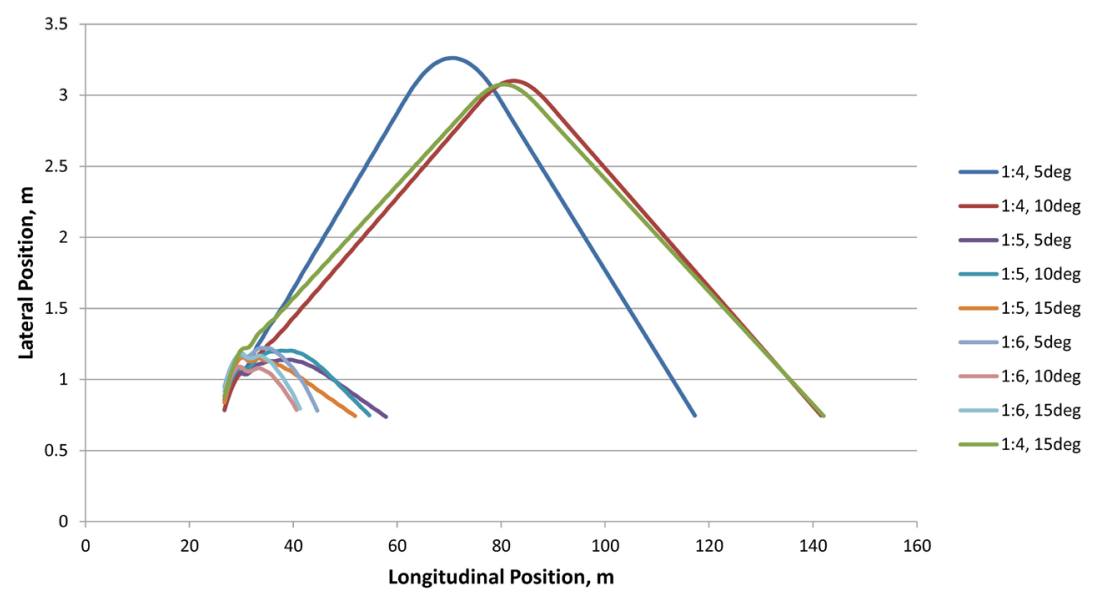

Figure 4. Overlapped vehicle trajectory after collision-Class C Hatchback on three median profiles.

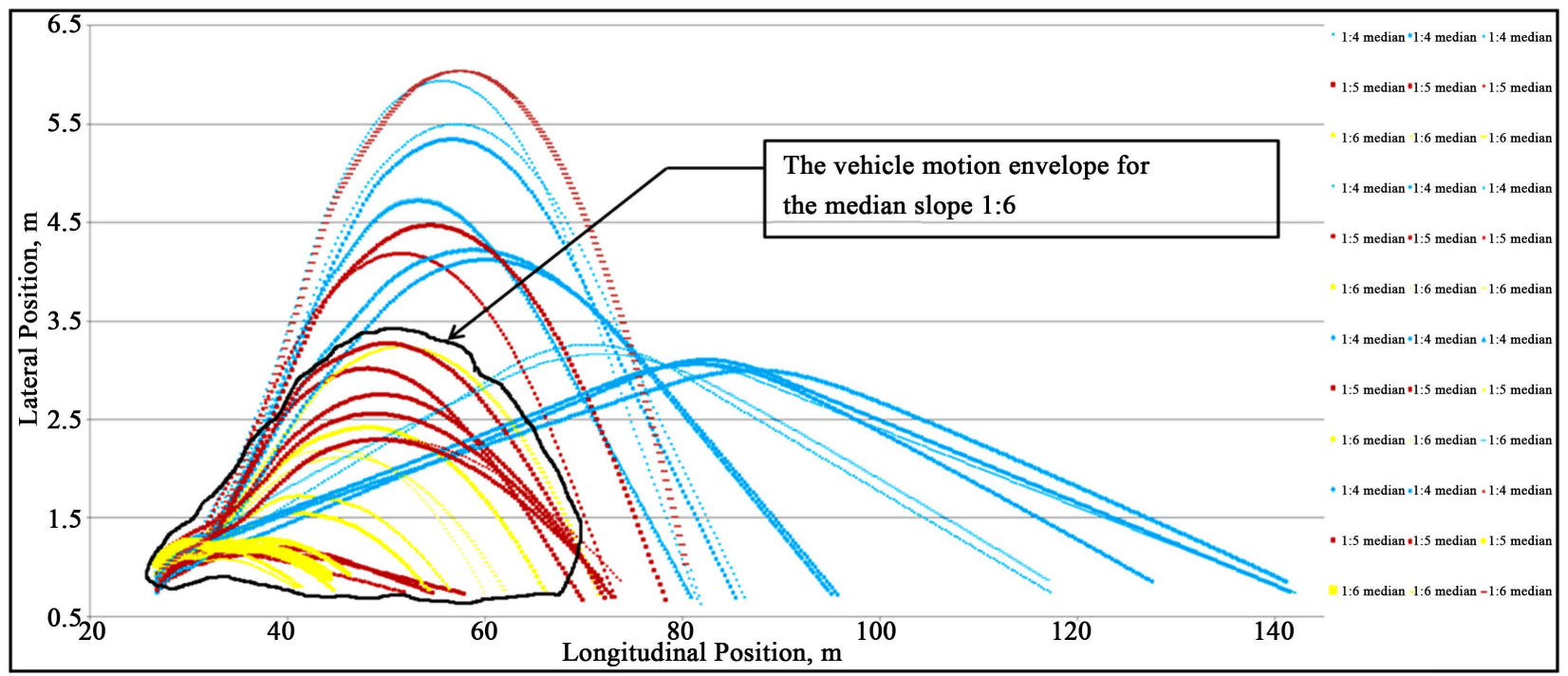

Figure 5. Overlapped trajectory of vehicle after collision-all vehicles on three median profiles. 
Table 2. Average maximum displacement of vehicle after the collision for three median profiles.

\begin{tabular}{cccc}
\hline Median Type & $1: 4$ median & $1: 5$ median & $1: 6$ median \\
Parameters & 4.1222 & 2.8520 & 1.7076 \\
Average maximum lateral displacement $(\mathrm{m})$ & 1.2634 & 1.2634 & 0.6569 \\
\hline
\end{tabular}

\section{Conclusions}

This study investigates the use of a sloped road median with a containment barrier to prevent an errant vehicle from being redirected into the path of other vehicles. A time-saving simulation method has been developed for assessing the effectiveness of different median slopes in containing such errant vehicles within the sloped median. A simple collision model based on planar impact mechanics has been implemented to simulate the collision of the vehicle with the containment barrier, greatly reducing the computational cost associated with the collision phase of the errant vehicle's trajectory in comparison with other methods such as Finite Element analysis. This model is implemented in MATLAB ${ }^{\mathrm{TM}}$ and works in conjunction with CarSim $^{\mathrm{TM}}$ to simulate the trajectory of the errant vehicle. The total time for simulating one errant vehicle sloped median encroachment including pre- and post-collision simulation in CarSim $^{\mathrm{TM}}$ plus collision in Matlab $^{\mathrm{TM}}$ is less than 30 seconds.

By comparing vehicle $x-y$ trajectories for median profiles with each other for different encroachment angles, the 1:6 median slope was shown to be the most effective one in containing the errant vehicle. Future study is anticipated to include 4 DOF vehicle collision models, which could predict the post-collision roll motion, in the simulation method, and to validate the simulation method by comparing the simulation results with the result for the same vehicle situation generated by professional vehicular collision software, EDSMAC 4 or PC-crash.

\section{References}

[1] New Jersey Department of Transportation Bureau of Research and US Department of Transportation Federal Highway Administration. Technical Report, February 2005. http://www.sbes.vt.edu/gabler/publications/Reports/CrossMedian-NJ-2005-04.pdf

[2] The National Crash Analysis Center. Working Paper, February 2011.

[3] The National Crash Analysis Center. Working Paper, August 2010.

[4] The National Crash Analysis Center. Technical Summary, September 2008.

[5] The National Crash Analysis Center. Working Paper, November 2009.

[6] Stine, J.S., Hamblin, B.C., Brennan, S.N. and Donnell, E.T. (2010) Analyzing the Influence of Median Cross-Section Design on Highway Safety Using Vehicle Dynamics Simulations. Accident Analysis and Prevention, 42, 1769-1777. https://doi.org/10.1016/j.aap.2010.04.018

[7] Uzunsoy, E., Bolarinwa, E., Olatunbosun, O. and He, R. (2014) Investigating Vehicle Behavior on a Sloped Terrain Surface. SAE Technical Paper 2014-01-0857. https://doi.org/10.4271/2014-01-0857 
[8] Brach, R.M. and Brach, M.R. (2005) Vehicle Accident Analysis and Reconstruction Methods. SAE International, Warrendale, PA.

[9] Zhou, J., Peng, H. and Lu, J.B. (2008) Collision Model for Vehicle Motion Prediction after Light Impacts. Vehicle System Dynamics, 46, 3-15.

[10] Day, T.D. (1999) An Overview of the EDSMAC4 Collision Simulation Model. SAE Technical Paper 1999-01-0102. SAE Publication SP-1407. Accident Reconstruction: Technology and Animation IX. Proceedings of the 1999 SAE International Congress \& Exposition, Detroit, MI, 1-4 March 1999. https://doi.org/10.4271/1999-01-0102

[11] Steffan, H. and Moser, A. (1996) The Collision and Trajectory Models of PC-CRASH. SAE Technical Paper 960886. https://doi.org/10.4271/960886

[12] Fay, R., Robinette, R., Scott, J. and Fay, P. (2001) PC-Crash and HVE, an Overview of Similarities and Differences. SAE Technical Paper 2001-01-0505. https://doi.org/10.4271/2001-01-0505

[13] Kuzel, M., Werner, S. and Richards, D. (2006) The Effect of Stiffness Coefficients on Output Variables in EDSMAC4 Simulations. SAE Technical Paper 2006-01-1396. https://doi.org/10.4271/2006-01-1396

[14] Hermann, S. (2006) PC-Crash Technical Manual. Version 7.3, Dr. Steffan Datentechnik GmbH., Linz, Austria.

[15] Prochowski, L. (2010) Analysis of Displacement of a Concrete Barrier on Impact of a Vehicle. Theoretical Model and Experimental Validation. Journal of KONES, 17, 399-406.

[16] Liu, W., et al. (2009) Springback Control of Sheet Metal Forming Based on the Response-Surface Method and Multi-Objective Genetic Algorithm. Materials Science and Engineering: $A, 499,325-328$. https://doi.org/10.1016/j.msea.2007.11.121

[17] Schram, R. and Geers, M.G.D. Accident Analysis and Evaluation of PC-Crash. http://www.mate.tue.nl/mate/pdfs/5707.pdf

[18] Brach, R.M. (2007) Mechanical Impact Dynamics: Rigid Body Collisions. Brach Engineering, LLC, Naperville, IL.

[19] New York State Department of Transportation (2013) Highway Design Manual. Chapter 2, Design Criteria. Revision 76, Limited Version, Albany, NY, 24.

[20] Lynam, D.A. and Kennedy, J.V. (2005) The Travel of Errant Vehicles after Leaving the Carriageway. Transport Research Laboratory (TRL), Wokingham.

[21] Plaxico, C.A. (2005) Recommended Guidelines for Curb and Curb-Barrier Installations. Vol. 537, Transportation Research Board, Washington.

[22] Marzougui, D., et al. (2010) Using Vehicle Dynamic Simulation as a Tool for Analyzing Cable Barrier Effectiveness. Tech. Rep. NCAC 2010-W-006. National Crash Analysis Center, Ashburn, VA.

[23] Mechanical Simulation (2012) Vhicle Shapes Provided with CarSim. https://carsim.com/downloads/pdf/CarSim_Vehicle_Catalog.pdf

[24] Gale, W.F. and Terry, C. (2003) Smithells Metals Reference Book. Butterworth-Heinemann, Oxford.

[25] Lapapong, S. (2011) Vehicle Rollover Prediction for Banked Surfaces. The Pennsylvania State University, State College, PA. 